\title{
Quantum Transport in Crystalline Semiconductors
}

\author{
One of the major topics discussed at the 3rd. EPS Condensed \\ Matter Division Conference on Electron Transport and Molecular \\ Solids held in Leeds at the end of July.
}

Quantum transport in solids can be defined as the flow of charge or of heat under conditions where the movement of the quasi-particles transporting energy through the solid cannot be described at all accurately by classical equations of motion such as the familiar Boltzmann equation. Such conditions may arise when the separation of individual quantum levels becomes comparable with, or greater than, the thermal energy of the carriers or when the particle wavelength becomes of the same order as the sample dimensions or of the physical size of the scattering centres. In this regime, various new effects become observable which are explicable only by a treatment which specifically includes the quantummechanical nature of the system. This paper reviews a number of recently discovered quantum transport phenomena. The review will be confined to effects observable in the d.c. electrical resistance of solids where abrupt changes can be induced by changing an externally applied parameter such as the magnetic or electric field. The sharpness of the structure generated by quantum effects is often such, that a similar precision is achieved in simple d.c. electrical measurements to that obtained when the system is studied spectroscopically with high frequency electromagnetic radiation. Thus, contrary to the normal expectancy of spectroscopists that an increase of the measuring frequency improves the experimental resolution, d.c. quantum transport measurements are competitive in precision with high frequency techniques such as cyclotron resonance in determining important parameters such as the effective mass of the charge carriers. Consequently zero must be considered to be a significant and accurate spectroscopic frequency.

\section{The Magnetophonon Effect}

A phenomenon which can be explained only by consideration of the quantisation of the phonon spectrum in addition to that of the electronic system is the magnetophonon effect first predicted by Gurevich and Firsov '). Because of the limited range in wavevector $k$ of a classical distribution of carriers, the high energy phonons or phonon combinations which contri- bute to scattering have a single welldefined frequency $\left(\omega_{p}\right)$. When the phonon energy equals the separation between any two Landau levels, i.e. when $h \omega_{p}=N h \omega_{c^{\prime}}$ the scattering of the carriers has a maximum value, and corresponding peaks periodic in $1 / B$ can be detected in the electrical resistance of the sample. Usually the phonon energy involved is known very accurately from Raman scattering experiments. Consequently one of the main applications of the magnetophonon effect has been a simple and precise method of determining the effective mass of the charge carriers in a variety of semiconductors. When a multivalley band structure exists, intervalley scattering by phonons of large wavevector can occur in addition to intravalley scattering by zone-centre phonons.

The type of phonons involved in intervalley scattering has long been controversial even with the exhaustively investigated elemental semiconductors, silicon and germanium. Because of the many different phonon energies involved, the magnetophonon series observed with silicon are extremely complex and the exact frequencies concerned are only revealed by Fourier analysis ${ }^{2}$ ). Such analysis has shown that at least five different types of phonon contribute to intervalley scattering and has helped to resolve the controversy concerning their nature. Another extremely successful application of the magnetophonon effect has been in the investigation of non-equilibrium or 'hot' carrier systems at low temperatures where a number of new processes for energy relaxation have been discovered. These include the emission of pairs of phonons located at the zone boundary, capture at an impurity site accompanied by L.O. phonon emission, and the 'magneto-impurity' effect where one of the quantised energies of the impurities replaces the phonon energy in the magnetophonon resonance condidition. In all cases the precision inherent in the determination of effective mass, ?honon energy or threshold energy for energy relaxation is of the order of $1 \%$ so that spectroscopic accuracy is achieved even though only simple d.c. techniques of measurement are involved.

\section{Quantisation Produced by Confinement of Electronic Wavefunctions}

Size quantisation arises from the wave nature of the carriers when either the potential barriers produced by the physical boundaries of the sample, or a spatially varying potential within the sample, confines the particles to a sufficiently small region of space. With high purity material, discrete quantum levels become resolved when the confinement distance is reduced below $1000 \AA$. When the kinetic energy of the particles is less than, or comparable with, the spacing of the size quantised levels, the motion in all directions of confinement is restricted, leading to a loss of dimensionality in transport properties. In this case, the particles cannot easily transfer between levels and quantum transport effects can be observed. When the confinement only occurs in one direction, as is the case in a thin film, the system is said to be two-dimensional.

As a result of the extraordinary control achieved by molecular beam epitaxial methods of growth, it is now possible to prepare periodic structures of complex geometry with extremely well defined dimensions along the growth direction. The resultant superlattice causes the electronic bands to break up into sub-bands because of the formation of additional Brillouin zones with generation of non-linear effects for electronic motion along the direction of the superlattice; see the review by Dingle ${ }^{3}$ ).

A further intriguing example of size quantisation, in this case produced by the combination of a surface and a spatially varying potential, is provided by the trapping of electrons outside the surface of liquid helium where the electron is bound by its own image charge within the helium itself ; see the review by $\mathrm{Cole}^{4}$ ). As the dielectric constant of liquid helium is only slightly greater than unity, the image potential is small and the electron is bound in a series of levels resembling those of the hydrogen atom but whose ground state energy is only $0.6 \mathrm{meV}$. Consequently hydrogenlike transitions between the bound state can be observed in the microwave région. Although the electrons 
are actually localised in a vacuum, they show many of the characteristics of carriers in a solid as far as their conduction processes are concerned.

An analogous system, where the confinement is also produced by an interface and an electrostatic potential, is provided by the carriers in the space charge layer at the semiconductor-insulator interface in MetalOxide-Semiconductor (MOS) structures. In MOS devices, the carriers are induced at the surface of the semiconductor by means of a voltage of opposite polarity applied to the metal gate forming one plate of the MOS capacitance structure. A solution of Poisson's equation for the space charge region shows that the carrier wavefunctions typically only extend a distance of the order of ten atomic spacings from the interface. Consequently the separation between the ground and first excited states is greater than $10 \mathrm{meV}$ for only moderate densities of surface charge. Thus, at low temperatures, the carriers are almost completely confined to the ground state and two-dimensional behaviour results. A unique feature of the MOS structure is that, by simply varying the voltage applied to the gate, the Fermi energy can be moved from outside the band concerned, up to an energy approaching $100 \mathrm{meV}$. This experimental feature, combined with the two-dimensional nature of the space-charge layer, have made the MOS device a particularly interesting system for fundamental research in addition to its widespread applications in technology.

One example of the significance of MOS structures for fundamental research concerns the theory of conduction in disordered systems. Provided that the disorder is not too great, the states at the centre of the band remain of high mobility and extended in character but the wavefunctions at the edge of the band lose phase-coherence very rapidly and become localized in nature ${ }^{5}$ ). A critical energy exists where the mobility drops sharply in value. By varying the gate voltage, the mobility on either side of this "mobility edge" can readily be explored. When the Fermi level lies at the critical energy and the temperature is low so that there is little thermal excitation of the carriers, the conductivity of a two-dimensional system should approach a value of $\cong 2 \pi e^{2} / \mathrm{h}$ which is independent of any scattering parameter or the band structure of the material concerned. In contrast the conductivity of a three dimensional system under the same conditions should be $\cong\left(2 \pi e^{2} / h\right) / \lambda$. where $\lambda$ is a rather ill-defined scattering length related to the scale of the random potential fluctuations. The three dimensional result cannot be checked precisely because of theoretical and experimental limitations in estimating $\lambda$ but the two dimensional result has been verified with MOS devices having widely different amounts of disorder ${ }^{6}$ ).

The Shubnikov-de Haas effect observed with space charge layers is very much modified by the two-dimensional nature of the gas. With a threedimensional bulk sample, a magnetic field only quantises the motion in the plane perpendicular to the field and leaves the motion along the field unquantised. As a result of this remaining unquantised degree of freedom, it is unusual with bulk samples to see more than a few percent change in resistance, as each Landau level successively empties on increasing the field. In contrast, complete quantisation is achieved when a magnetic field is applied perpendicular to a space charge layer. Thus, even though the mobilities available are not in general as high as with bulk material, the amplitude of the Shubnikov-de Haas effect observed with MOS structures can be very high, and resistance swings as great as $10^{7}$ have been observed. The large amplitude of the effect can be understood very simply. If the charge density induced in the surface is just sufficient to fill $n$ Landau levels and the $n+1$ level is completely empty, then no conduction should occur because of the finite energy required to excite a carrier, and the device is effectively an insulator. If, however, a Landau subband is only half-full, a metallic type of conduction can occur.

On the simplest model outlined above, the resistivity should become infinite when the Fermi energy lies half-way between adjacent Landau sub-bands. In practice, however, the resistance remains finite either because of thermal excitation between Landau levels, which is analogous to intrinsic excitation between valence and conduction bands in a normal semiconductor, or because of overlap between the states in the tails of the Landau levels. In the latter case, conduction can proceed either as with a semimetal if the states are extended, or through a thermally activated process, as with an amorphous semiconductor, if the tail states are localised in character. From a detailed analysis of the temperature and field dependence of the conductivity minima, "intrinsic" "semi-metallic" and "amor- phous" conduction have all been detected experimentally ${ }^{7}$ ).

In addition to this behaviour which can all be discussed in terms of single particle excitations, a number of features are observed experimentally which are only explicable by co-operative phenomena. The peak conductivity which occurs when a Landau level is half-full, is found to be thermally activated at high magnetic fields, whereas it is expected to be independent of temperature, scattering and effective mass of the charge carrier, taking a value of approximately $\left(2 \pi e^{2} / h\right)(n+1 / 2)$. The valley degeneracy of the silicon conduction band deduced from the period of the Shubnikov - de Haas resistance maxima is often lower than that expected for the particular surface concerned and the cyclotron effective masses are frequently a function of the gate voltage and of externally applied stress. The apparent change in the band structure has been explained in terms of the existence of charge or spin density waves which introduce symmetrybreaking distortions and lower the total energy of the system ${ }^{8}$ ).

The thermal activation of the conductivity maxima may arise either from excitation above a mobility edge associated with the disorder at the semiconductor-insulator interface or from "Wigner crystallisation" where the electron gas as a whole becomes ordered at low temperatures. The relative importance of density waves, Wigner crystallisation and disorderinduced effects in real systems has not yet been established. All of these possibilities are significantly modified by the two-dimensional nature of the space-charge layers. Consequently, quantum transport in MOS structures will be studied intensively during the coming years in order to define the factors which determine the dominance of any one of these competing effects.

\section{Further Reading}

1. GUREVICH, V.L. and FIRSOV, Y.A. Sov. Phys. JETP 13 (1961) 137

2. EAVES, L., HOULT, R.A., STRADLING, R.A., TIDEY, R.J., PORTAL, J.C. and ASKENAZY, S. J. Phys. C8 1034

3. DINGLE, R., Festkorperprobleme XV Adv. in Solid State Physics (1975), p. 21

4. COLE, M.W. Rev. Mod. Phys. 46 (1974) 451

5. ANDERSON, P.W. Phys. Rev. 109 (1958) 1492

6. MOTT, N.F., PEPPER, M., POLLITT, S., WALLIS, R.H. and ADKINS, C.I. Proc. Roy. Soc (London) A345 (1975) 169

7. NICHOLAS, R.J., STRADLING, R.A. and TIDEY, R.J. Solid State Comm. (1977)

8. KELLEY, M.J. and FALIVOC, L.M. Phys. Rev. B15 (1977) 1974 